\title{
An effective thermal conductivity model for fractal porous media with rough surfaces
}

\author{
Xuan Qin ${ }^{1,2}$, Yingfang Zhou ${ }^{2} *$, Agus Pulung Sasmito ${ }^{3}$ \\ ${ }^{1}$ Hubei Subsurface Multi-scale Imaging Key Laboratory, Institute of Geophysics and Geomatics, \\ China University of Geosciences, Wuhan 430074, P. R. China \\ ${ }^{2}$ School of Engineering, University of Aberdeen, Aberdeen, AB24 3UE, UK \\ ${ }^{3}$ Department of Mining and Materials Engineering, McGill University, 3450 University Street, \\ Montreal, QC H3AOE8, Canada
}

(Received January 12, 2019; revised January 28, 2019; accepted January 29, 2019; available online February 8, 2019)

\section{Citation:}

Qin, X., Zhou, Y., Sasmito, A.P. An

effective thermal conductivity model for

fractal porous media with rough surfaces.

Advances in Geo-Energy Research, 2019,

3(2): 149-155, doi:

10.26804/ager.2019.02.04.

Corresponding author:

*E-mail: yingfang.zhou@abdn.ac.uk

Keywords:

Effective thermal conductivity

porous media

rough surfaces

fractal

\begin{abstract}
:
Quantitative evaluation of the effective thermal conductivity of porous media has received wide attention in science and engineering since it is a key thermophysical parameter in characterizing heat transfer properties. Based on fractal characters of tortuous capillary tubes and rough surfaces in micro-pores, we proposed a theoretical model of the effective thermal conductivity in porous media with rough surfaces. This model considers the geometrical parameters of porous media, including porosity, micro-pore fractal dimension, tortuosity fractal dimension, and relative roughness. The calculated normalized effective thermal conductivity was then validated against published experimental data. The results show good agreement between them. The influence of geometrical factors, porosity and relative surface roughness, on the effective thermal conductivity in porous media with rough surfaces are discussed and analyzed extensively.
\end{abstract}

\section{Introduction}

The effective thermal conductivity of porous media is an important property in many engineering and science applications, such as geothermal energy (Clauser, 2009), petroleum engineering (Dai et al., 2015), climate change (Kooi, 2008), and nuclear wastes (Jougnot and Revil, 2010). The effective thermal conductivity of heterogeneous porous medium is affected by the complicated relationship between the topology and geometry of pore spaces and solid matrix (Sahimi, 2011; Ghanbarian and Daigle, 2016). Thus, many effective thermal conductivity models have been proposed based on classical mixing laws. The upper and lower limits of thermal conductivity for two-phase saturated porous media corresponding to the well-known weighted arithmetic and harmonic means are generally defined by the parallel and series models (Clauser, 2009; Ranut, 2016). The Maxwell-Eucken model is often used to characterize the thermal conductivity of porous media, where spherical pores are assumed to be widely dispersed in a continuous medium (Carson et al., 2005). The effective medium theory (EMT) is an approximated method used to model the thermal conductivity of the macroscopically homogeneous and isotropic media containing randomly distributed grains and pores (Revil, 2000; Sadeghi et al., 2018). The hybrid method is another approach to evaluate the effective thermal conductivity of porous media consisting of five basic structural models (Wang et al., 2006; Behrang et al., 2016). However, it is somewhat challenging to determine appropriate parameters for this method. In recent decades, the fractal theory has been used to study the effective thermal conductivity of porous media to account for the effect of porous media's microstructure. Yu and Cheng (2002a) proposed an effective thermal conductivity of bidispersed porous media based on fractal and thermal-electrical analogy theory. Xu et al. (2006) analyzed the effective thermal conductivity of the symmetric 


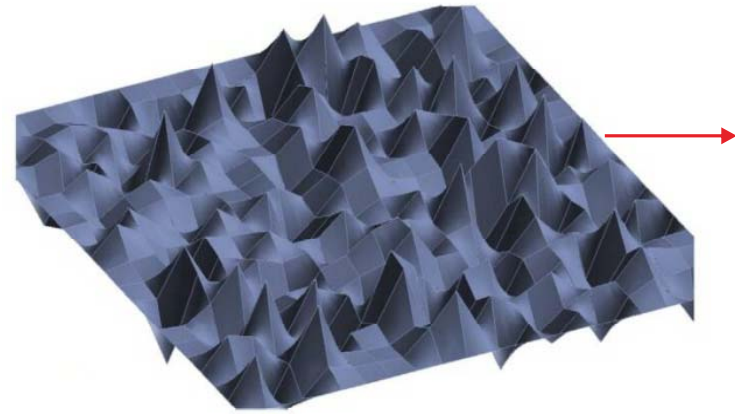

(a)

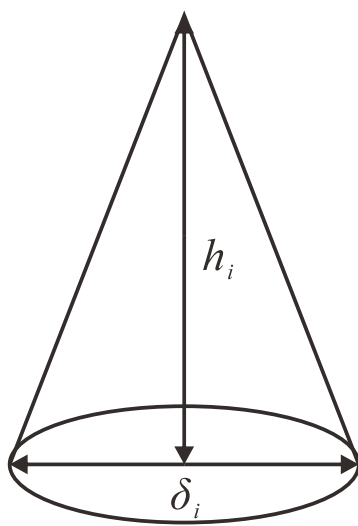

(b)

Fig. 1. Schematic illustrations of the rough surface. (a) A real surface with the cone-shaped rough elements (Guo et al., 2015); (b) A representative cone-like rough element, where $h$ is the height of a cone-shaped element and $\delta$ is the base diameter of a cone-shaped element.

fractal tree-like branched networks. Pia and Sanna (2014) and Pia et al. (2016) presented an intermingled fractal model to predict the effective thermal conductivity. Shen et al. (2017) proposed an anisotropic fractal model to evaluate the effective thermal conductivity of the random metal fiber porous media. Qin et al. (2019) proposed a theoretical fractal model of effective thermal conductivity in porous media with various liquid saturation. In addition to these theoretical models, many numerical approaches such as Monte Carlo method (Wang and $\mathrm{Li}, 2017$ ), finite element method (Li et al., 2017), and lattice Boltzmann method (Wang et al., 2007) have also been adopted to study the thermal conductivity of porous media. However, the impact of pore surface roughness has not been incorporated by mentioned above model to simulate the effective thermal conductivity.

The pore surfaces in natural porous media, in general, are rough; and the impact of roughness on thermal conductivity has been investigated extensively, primary through heat transfer measurements. Tang et al. (2007) investigated the fluid flow in stainless steel and fused silica micro-tubes experimentally. Their results indicated that the effects of surface roughness in micro-channels could not be neglected if relative surface roughness is larger than $1 \%$. Another experiment study was carried out by $\mathrm{Li}$ et al. (2007) to measure the flow and heat transfer characteristics of the liquid in the smooth and rough micro-pores. They concluded that the conventional friction theory might be not useful in rough micro-pores. Majumdar and Bhushan (1990) observed that the fractal theory had the potential to describe the behavior of rough surface phenomenon. Chen and Zhang (2014) introduced the fractal Cantor structure to characterize the rough surfaces and proposed a molecular dynamics simulation to study the impact of rough surfaces on heat conduction in micro-channels. Guo et al. (2015) developed the 2D roughness model and 3D Gauss model to disclose the fluid flow and heat transfer in micro-channels with roughness. They found that both flow performance and thermal transport process were sensitive to the surface morphology. Askari et al. (2017) presented a numerical simulation of heat conduction in a packing of particles with rough self-affine fractal surfaces.

To the best of our knowledge, the analytical expression of effective thermal conductivity in porous media with rough surfaces is rarely reported. In this study, a theoretical effective thermal conductivity model of porous media is proposed based on fractal characters of tortuous capillary tubes and rough surfaces in micro-pores. The proposed fractal model is then validated by published experimental data, and the effect of various geometrical parameters on the effective thermal conductivity of porous media are discussed in detail.

\section{A fractal thermal conductivity model for porous media with rough surfaces}

\subsection{Fractal characteristics of rough surfaces}

Some researches (Zou et al., 2008; Cai et al., 2010; Yang et al., 2014; Guo et al., 2015) showed that the rough surfaces can be described as the cone-shaped rough elements. In this study, we also assume the rough surfaces are cone-shaped and every conic peak do not overlap each other (Fig. 1). Additionally, it has been shown that the rough surfaces follow the fractal scaling law (Mahovic Poljacek et al., 2008). Thus, the base size distribution of cone-shaped elements is assumed to obey the following relationship with base diameters from $\delta$ to $\delta+$ $d \delta$ (Yu et al., 2009):

$$
-d N=D \delta_{\max }^{D} \delta^{-(D+1)} d \delta
$$

where $\delta_{\max }$ is the maximum base diameter, $D$ is the base area fractal dimension, and $d N<0$ implies that the number of bases decreases with increasing of the base diameter.

The base area fractal dimension $D$ can be expressed as (Yu et al., 2009):

$$
D=D_{E}-\frac{\ln \varphi}{\ln \alpha}
$$

where $D_{E}$ is the Euclidean dimension $\left(D_{E}=2\right.$ is assumed in this study); $\varphi$ is the ratio of the total base area of cone-shaped 


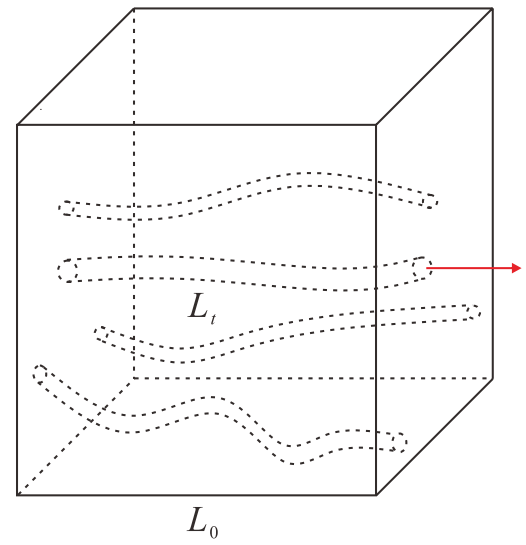

(a)

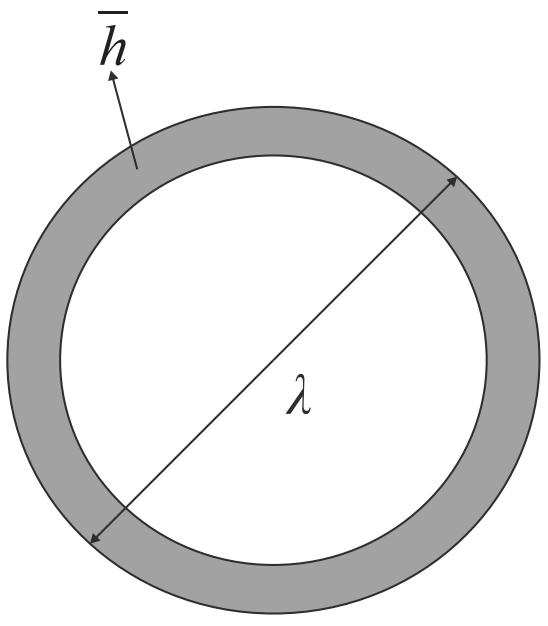

(b)

Fig. 2. (a) The schematic illustration of the porous media composed of a bundle of tortuous micro-pores; (b) The schematic of the cross-section of a micro-pore with rough surfaces, where $\lambda$ is the micro-pore diameter and $\bar{h}$ is the average height of all cone-shaped elements in a micro-pore.

elements to the surface area of a micro-pore; $\alpha=\delta_{\min } / \delta_{\max }$; and $\delta_{\min }$ is the minimum base diameter.

As shown in Fig. 1, the base area of a cone-shaped element $S_{i}$ can be written as:

$$
S_{i}=\frac{\pi \delta_{i}^{2}}{4}
$$

The volume of a cone-shaped element $V_{i}$ can be expressed as:

$$
V_{i}=\frac{1}{3} S_{i} h_{i}=\frac{\pi \delta_{i}^{3}}{12} \zeta
$$

where $\zeta=h / \delta$ is the ratio of the height to the base diameter of a cone-shaped element.

Based on Eqs. (1) and (3), the total base area of all coneshaped elements $S$ can be obtained by an integration of all diameters from $\delta_{\min }$ to $\delta_{\max }$ :

$$
S=-\int_{\delta_{\min }}^{\delta_{\max }} S_{i} d N=\frac{\pi D \delta_{\max }^{2}(1-\varphi)}{4(2-D)}
$$

From Eqs. (1) and (4), the total volume of all cone-shaped elements $V_{t}$ can be written as:

$$
V_{t}=-\int_{\delta_{\min }}^{\delta_{\max }} V_{i} d N=\frac{\pi \zeta D \delta_{\max }^{3}\left(1-\alpha^{3-D}\right)}{12(3-D)}
$$

The surface area of a micro-pore $S_{t}$ can be expressed as:

$$
S_{t}=\frac{S}{\varphi}=\frac{\pi D \delta_{\max }^{2}(1-\varphi)}{4(2-D) \varphi}
$$

Combining Eqs. (6) and (7), the average height of all coneshaped (rough) elements $\bar{h}$ can be given as:

$$
\bar{h}=\frac{V_{t}}{S_{t}}=\frac{\zeta \varphi \delta_{\max }(2-D)\left(1-\alpha^{3-D}\right)}{3(3-D)(1-\varphi)}
$$

The porous media is often considered to be composed of a bundle of tortuous micro-pores (see Fig. 2), whose size distribution follows the fractal scaling law (Cai et al., 2014). Thus, the micro-pore diameter $\lambda$ distribution has a similar relationship with Eq. (1):

$$
-d N=D_{f} \lambda_{\max }^{D_{f}} \lambda^{-\left(D_{f}+1\right)} d \lambda
$$

where $\lambda_{\max }$ is the maximum micro-pore diameter, $D_{f}$ is the micro-pore fractal dimension.

The micro-pore fractal dimension $D_{f}$ also can be expresses as:

$$
D_{f}=D_{E}-\frac{\ln \phi}{\ln \beta}
$$

where $\phi$ is the porosity, $\beta=\lambda_{\min } / \lambda_{\max }$ and $\lambda_{\min }$ is the minimum micro-pore diameter.

The relative roughness $\gamma$ can be defined by (Yang et al., 2015):

$$
\gamma=\frac{2 \bar{h}}{\lambda}
$$

It is assumed that the micro-pores in porous media have identical relative roughness. Thus, Eq. (11) can be rewritten as:

$$
\frac{\left(h_{\max }\right)_{\min }}{\left(h_{\max }\right)_{\lambda}}=\frac{\lambda_{\min }}{\lambda}
$$

where $\left(h_{\max }\right)_{\lambda_{\min }}$ is the maximum height of cone-shaped element in the minimum micro-pore and $\left(h_{\max }\right)_{\lambda}$ is the maximum height of cone-shaped element in a micro-pore.

Inserting Eqs. (8) and (12) into Eq. (11), the relative roughness $\gamma$ can be rewritten as:

$$
\gamma=\frac{2 \varphi\left(h_{\max }\right) \lambda_{\min }(2-D)\left(1-\alpha^{3-D}\right)}{3 \lambda_{\min }(3-D)(1-\varphi)}
$$

Eq. (13) indicates that the relative roughness in the rough micro-pores is related to geometrical parameters. Thus, each parameter in Eq. (13) has a physical meaning. 


\subsection{Fractal model for effective thermal conductivity of porous media with rough surfaces}

Based on the classical Fourier's law and the thermalelectrical analogy method, the thermal resistance of a single tortuous capillary tube with rough surfaces $r_{f}$ can be expressed as (Miao et al., 2016):

$$
r_{f}=\frac{4 L_{t}}{\pi k_{f}(\lambda-2 \bar{h})^{2}}=\frac{4 L_{t}}{\pi k_{f} \lambda^{2}(1-\gamma)^{2}}
$$

where $L_{t}$ is the actual length of fluid path and $k_{f}$ is the thermal conductivity of fluid. The actual length of fluid path is given by (Yu and Cheng, 2002b):

$$
L_{t}=\lambda^{1-D_{T}} L_{0}^{D_{T}}
$$

where $L_{0}$ is the characteristic length along the direction of heat flow and $D_{T}$ is the tortuosity fractal dimension for micro-pore.

The relationship between the fractal dimension characteristic length $L_{0}$ and the total cross-sectional area $A$ can be expressed by:

$$
L_{0}=\sqrt{A}
$$

The total cross-sectional area $A$ can be determined by (Cai et al., 2012):

$$
A=\frac{\pi D_{f} \lambda_{\max }^{2}(1-\phi)}{4\left(2-D_{f}\right) \phi}
$$

The tortuosity fractal dimension for micro-pore can describe the tortuous degree of the capillary tubes in porous media quantitatively. This specific microstructural parameter of $D_{T}$ is difficult to determine experimentally, but can be calculated through an approximate formula (Wei et al., 2015):

$$
D_{T} \approx\left(D_{E}-D_{f}+1\right)+\left(D_{E}-D_{f}\right) \frac{\log D_{f}-\log \left(D_{f}-1\right)}{\log \phi}
$$

As the capillary tubes considered to be parallel, the total thermal resistance of fluid in rough micro-pores $R_{f}$ can be obtained as:

$$
\frac{1}{R_{f}}=-\int_{\lambda_{\min }}^{\lambda_{\max }} \frac{1}{r_{f}} d N
$$

Inserting Eqs. (9), (14), and (15) into Eq. (19), the total thermal resistance of fluid in rough micro-pores can be given as:

$$
R_{f}=\frac{4 L_{0}^{D_{T}}\left(D_{T}-D_{f}+1\right)}{\pi k_{f} D_{f} \lambda_{\max }^{D_{T}+1}\left(1-\phi^{\frac{D_{T}-D_{f}+1}{2-D_{f}}}\right)(1-\gamma)^{2}}
$$

The thermal resistance of solid matrix $R_{S}$ can be written as:

$$
R_{s}=\frac{L_{0}}{(1-\phi) A k_{s}}
$$

where $k_{s}$ is the thermal conductivity of solid matrix.
The effective thermal conductivity of porous media with rough surfaces $k_{e}$ can be expressed as:

$$
k_{e}=\frac{L_{0}}{R_{t} A}=\frac{L_{0}}{A}\left(\frac{1}{R_{f}}+\frac{1}{R_{s}}\right)
$$

Finally, based on Eqs. (16), (17), (20) and (21), Eq. (22) of the effective thermal conductivity of porous media with rough surfaces can be obtained as:

$$
\begin{aligned}
& k_{e}=\frac{k_{f}\left(2-D_{f}\right) \phi\left(1-\phi^{\frac{D_{T}-D_{f}+1}{2-D_{f}}}\right)\left(\frac{\lambda_{\max }}{L_{0}}\right)^{D_{T}-1}}{\left(D_{T}-D_{f}+1\right)(1-\phi)}(1-\gamma)^{2} \\
& +(1-\phi) k_{s}
\end{aligned}
$$

Eq. (23) is a function of porosity $(\phi)$, fractal dimension of the micro-pore $\left(D_{f}\right)$, tortuosity fractal dimension of the micro-pore $\left(D_{T}\right)$, characteristic length $\left(L_{0}\right)$, maximum micropore diameter $\left(\lambda_{\max }\right)$, relative roughness $(\gamma)$, and the thermal conductivity of the solid matrix and fluid. This proposed theoretical model reveals the physical basis of heat transfer in porous media with rough surfaces.

\section{Results and discussion}

In this section, we first compare the results from our proposed model with various experimental measurements from other available literature. Then, we discuss the sensitivity of the proposed model to their parameters, such as porosity and relative roughness.

Fig. 3 presents the comparison between the proposed model prediction and experimental data for saturated porous media with porosity of 0.4 (Woodside and Messmer, 1961; Prasad et al., 1989; Kaviany, 1995; Kou et al., 2009). Some researches (Feng et al., 2007; Miao et al., 2016) have found that the $\lambda_{\min } / \lambda_{\max }$ ratio is usually in the range of $10^{-2} \sim 10^{-4}$

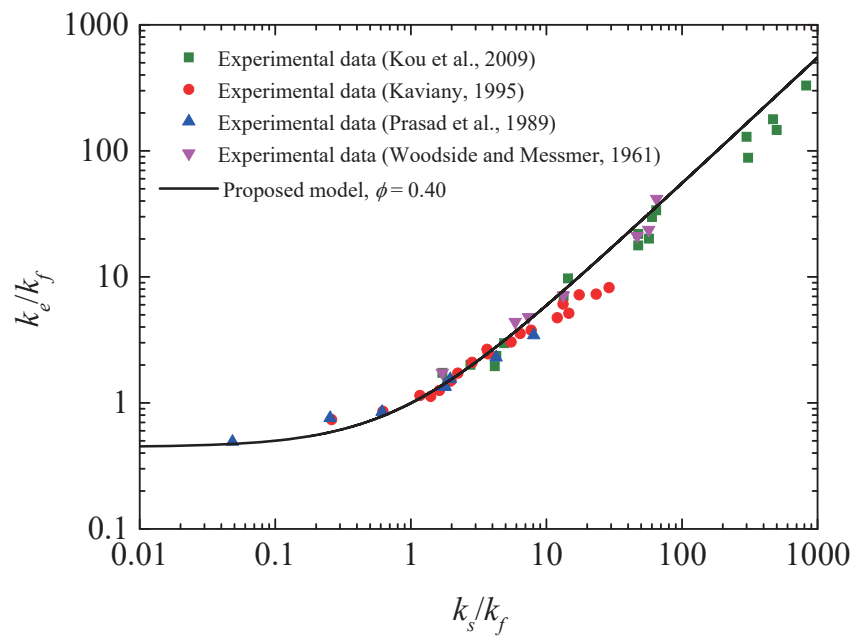

Fig. 3. The comparison between proposed model prediction and experimental data for saturated porous media when the porosity equals to 0.40 , where $\alpha=$ $0.01, \beta=0.01,2\left(h_{\max }\right) \lambda_{\min } / \lambda_{\min }=0.1$ and $\varphi=0.2$. 


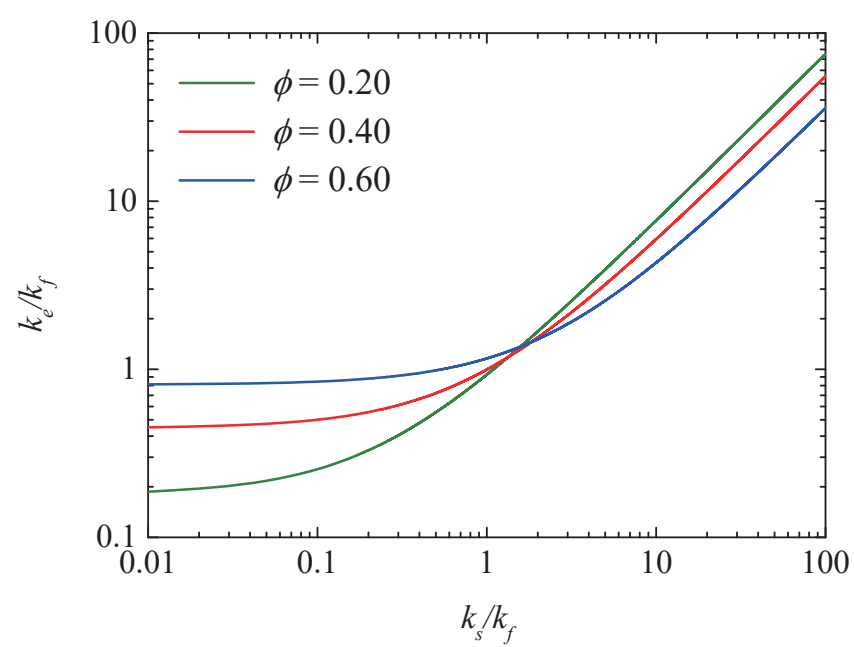

Fig. 4. The dimensionless effective thermal conductivity versus the thermal conductivity ratio of the solid phase to fluid phase at different porosity, where $\alpha=0.01, \beta=0.01,2\left(h_{\max }\right)_{\lambda_{\min }} / \lambda_{\min }=0.1$ and $\varphi=0.2$.

in many natural porous media. Thus, $\alpha=0.01$ and $\beta=$ 0.01 are applied to this study. As the maximum height of the cone-shaped element to the micro-pore diameter is in the range of $0.12 \sim 0.17$ (Li et al., 2003), $2\left(h_{\max }\right) \lambda_{\min } / \lambda_{\min }=0.1$ is assumed in this study. The ratio of the total base area of cone-shaped elements to the surface area of a micro-pore is $\varphi=0.2$ (Cai et al., 2014). As can be seen in Fig. 3, the proposed model prediction is in good agreement with the available experimental data.

Fig. 4 shows the dimensionless effective thermal conductivity versus the thermal conductivity ratio of the solid phase to the fluid phase at a different porosity. It is seen that the dimensionless effective thermal conductivity increases as the porosity decreases when the thermal conductivity ratio of the solid to fluid phases $k_{s} / k_{f}>1$. On the contrary, if $k_{s} / k_{f}<$ 1 , the dimensionless effective thermal conductivity increases with the increase of the porosity. This can be explained by the fact that the volume fraction of the solid phase with higher thermal conductivity increases, leading to an increase of the effective thermal conductivity when $k_{s} / k_{f}>1$. Whereas, when $k_{s} / k_{f}<1$, the result is opposite. These results are analogous to those given by Miao et al. (2016).

Fig. 5 illustrates the dimensionless effective thermal conductivity versus the thermal conductivity ratio of the solid phase to the fluid phase at a different relative roughness when the porosity equals 0.40 . It is observed that the dimensionless effective thermal conductivity decreases with the increasing the relative roughness. This may be attributed to the effect on the heat transfer in micro-pores as the increase of the thermal resistance which is consistent with the result given by Askari et al. (2017). In addition, it is found that the dimensionless effective thermal conductivity is slightly dependent on the relative roughness when $k_{s} / k_{f}>10$. It implies that the effect of surface roughness in micro-pores may be neglected if the thermal conductivity of solid matrix much larger than the con-

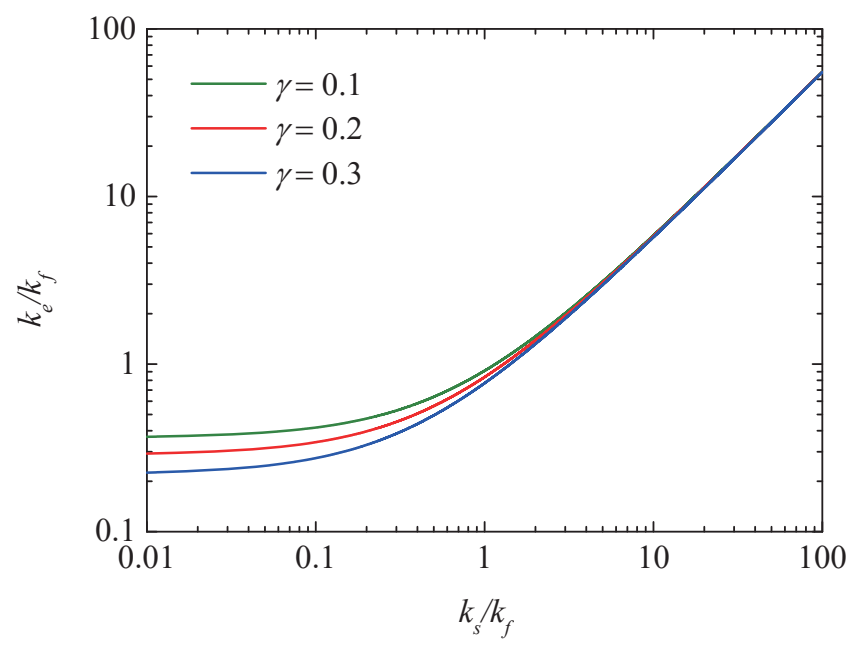

Fig. 5. The dimensionless effective thermal conductivity versus the thermal conductivity ratio of the solid phase to fluid phase at a different relative roughness when the porosity equals 0.40 .

ductivity of fluid.

\section{Conclusions}

In this work, the analytical expression for the effective thermal conductivity of porous media with rough surfaces is derived based on the fractal geometry theory. The effective thermal conductivity is a function of microstructural parameters, such as porosity, micro-pore fractal dimension, tortuosity fractal dimension for micro-pore, and relative roughness. The present model predictions show a good agreement with published experimental data of various natural and engineered materials. This newly proposed model allows the physical understanding of thermal transport in porous media with rough surfaces.

It should be noted that the theoretical model proposed in this study only focuses on the effect of the surface roughness in micro-pores and pore size distributions on the heat conduction in porous media. The model can be improved by considering the self-affine fractals in micro-pores and the varying relative roughness in different micro-pores. Also, thermal transport in natural porous media involves convection and radiation that has not been considered in this study.

\section{Acknowledgments}

This project was supported by the National Natural Science Foundation of China (No. 41572116), and the Hubei Provincial Natural Science Foundation of China (No. 2018CFA051). Dr. Zhou would like to thank the Royal Society to support his trip to China with the International Exchange Programme and Mr. Qin would like to thanks the British Council and China Scholarship Council to support his stay at the University of Aberdeen through the UK-China PhD Placement Programme.

Open Access This article is distributed under the terms and conditions of 
the Creative Commons Attribution (CC BY-NC-ND) license, which permits unrestricted use, distribution, and reproduction in any medium, provided the original work is properly cited.

\section{References}

Askari, R., Hejazi, S.H., Sahimi, M. Effect of deformation on the thermal conductivity of granular porous media with rough grain surface. Geophys. Res. Lett. 2017, 44(16): 8285-8293.

Behrang, A., Taheri, S., Kantzas, A. A hybrid approach on predicting the effective thermal conductivity of porous and nanoporous media. Int. J. Heat Mass Transf. 2016, 98: $52-59$.

Cai, J., Hu, X., Standnes, D.C., et al. An analytical model for spontaneous imbibition in fractal porous media including gravity. Colloid Surf. A-Physicochem. Eng. 2012, 414: 228-233.

Cai, J., Perfect, E., Cheng, C., et al. Generalized modeling of spontaneous imbibition based on Hagen-Poiseuille flow in tortuous capillaries with variably shaped apertures. Langmuir 2014, 30(18): 5142-5151.

Cai, J., Yu, B., Zou, M., et al. Fractal analysis of surface roughness of particles in porous media. Chin. Phys. Lett. 2010, 27(2): 157-160.

Carson, J.K., Lovatt, S.J., Tanner, D.J., et al. Thermal conductivity bounds for isotropic, porous materials. Int. J. Heat Mass Transf. 2005, 48(11): 2150-2158.

Chen, Y., Zhang, C. Role of surface roughness on thermal conductance at liquid-solid interfaces. Int. J. Heat Mass Transf. 2014, 78: 624-629.

Clarkson, C.R., Solano, N., Bustin, R.M., et al. Pore structure characterization of North American shale gas reservoirs using USANS/SANS, gas adsorption, and mercury intrusion. Fuel 2013, 103(1): 606-616.

Clauser, C. Heat transport processes in the Earth's crust. Surv. Geophys. 2009, 30(3): 163-191.

Dai, S., Cha, J.H., Rosenbaum, E.J., et al. Thermal conductivity measurements in unsaturated hydrate-bearing sediments. Geophys. Res. Lett. 2015, 42(15): 6295-6305.

Feng, Y., Yu, B., Zou, M., et al. A generalized model for the effective thermal conductivity of unsaturated porous media based on self-similarity. J. Porous Media 2007, 10(6): 551-567.

Ghanbarian, B., Daigle, H. Thermal conductivity in porous media: Percolation-based effective-medium approximation. Water Resour. Res. 2016, 52(1): 295-314.

Guo, L., Xu, H., Gong, L. Influence of wall roughness models on fluid flow and heat transfer in microchannels. Appl. Therm. Eng. 2015, 84: 399-408.

Jougnot, D., Revil, A. Thermal conductivity of unsaturated clay-rocks. Hydrol. Earth Syst. Sci. 2010, 14(1): 91-98.

Kaviany, M. Conduction Heat Transfer in Principles of Heat Transfer in Porous Media. New York, USA, Springer Science \& Business Media, 2012.

Kooi, H. Spatial variability in subsurface warming over the last three decades; insight from repeated borehole temperature measurements in The Netherlands. Earth Planet. Sci. Lett. 2008, 270(1): 86-94.
Kou, J., Wu, F., Lu, H., et al. The effective thermal conductivity of porous media based on statistical selfsimilarity. Phys. Lett. A 2009, 374(1): 62-65.

Li, B., Xu, W., Tong, F. Measuring thermal conductivity of soils based on least squares finite element method. Int. J. Heat Mass Transf. 2017, 115(Part B): 833-841.

Li, Z., Du, D., Guo, Z. Experimental study on flow characteristics of liquid in circular microtubes. Microsc. Therm. Eng. 2003, 7(3): 253-265.

Li, Z., He, Y., Tang, G., et al. Experimental and numerical studies of liquid flow and heat transfer in microtubes. Int. J. Heat Mass Transf. 2007, 50(17): 3447-3460.

Majumdar, A., Bhushan, B. Role of fractal geometry in roughness characterization and contact mechanics of surfaces. J Tribol. 1990, 112(2): 205-216.

Miao, T., Cheng, S., Chen, A., et al. Analysis of axial thermal conductivity of dual-porosity fractal porous media with random fractures. Int. J. Heat Mass Transf. 2016, 102: 884-890.

Pia, G., Corcione, C.E., Striani, R., et al. Thermal conductivity of porous stones treated with UV light-cured hybrid organic-inorganic methacrylic-based coating. Experimental and fractal modeling procedure. Prog. Org. Coat. 2016, 94: 105-115.

Pia, G., Sanna, U. An intermingled fractal units model to evaluate pore size distribution influence on thermal conductivity values in porous materials. Appl. Therm. Eng. 2014, 65(1-2): 330-336.

Poljacek, S.M., Risovic, D., Furic, K., et al. Comparison of fractal and profilometric methods for surface topography characterization. Appl. Surf. Sci. 2008, 254(11): 34493458.

Prasad, V., Kladias, N., Bandyopadhaya, A., et al. Evaluation of correlations for stagnant thermal conductivity of liquidsaturated porous beds of spheres. Int. J. Heat Mass Transf. 1989, 32(9): 1793-1796.

Qin, X., Cai, J., Xu, P., et al. A fractal model of effective thermal conductivity for porous media with various liquid saturation. Int. J. Heat Mass Transf. 2019, 128: 11491156.

Ranut, P. On the effective thermal conductivity of aluminum metal foams: Review and improvement of the available empirical and analytical models. Appl. Therm. Eng. 2016, 101: 496-524.

Revil, A. Thermal conductivity of unconsolidated sediments with geophysical applications. J. Geophys. Res. 2000, 105(B7): 16749-16768.

Sadeghi, M., Ghanbarian, B., Horton, R. Derivation of an explicit form of the percolation-based effective-medium approximation for thermal conductivity of partially saturated soils. Water Resour. Res. 2018, 54(2): 13891399.

Sahimi, M. Flow and Transport in Porous Media and Fractured Rock: From Classical Methods to Modern Approaches. New Jersey, USA, John Wiley \& Sons, 2011.

Shen, H., Ye, Q., Meng, G. Anisotropic fractal model for the effective thermal conductivity of random metal fiber porous media with high porosity. Phys. Lett. A 2017, 
381(37): 3193-3196.

Tang, G., Li, Z., He, Y., et al. Experimental study of compressibility, roughness and rarefaction influences on microchannel flow. Int. J. Heat Mass Transf. 2007, 50(11): 2282-2295.

Wang, F., Li, X. The stagnant thermal conductivity of porous media predicted by the random walk theory. Int. J. Heat Mass Transf. 2017, 107: 520-533.

Wang, J., Carson, J.K., North, M.F., et al. A new approach to modelling the effective thermal conductivity of heterogeneous materials. Int. J. Heat Mass Transf. 2006, 49(17-18): 3075-3083.

Wang, M., Wang, J., Pan, N., et al. Mesoscopic predictions of the effective thermal conductivity for microscale random porous media. Phys. Rev. E 2007, 75(3): 036702.

Wei, W., Cai, J., Hu, X., et al. An electrical conductivity model for fractal porous media. Geophys. Res. Lett. 2015, 42(12): 4833-4840.

Woodside, W., Messmer, J.H. Thermal conductivity of porous media. I. Unconsolidated sands. J. Appl. Phys. 1961, 32(9): 1688-1706.
$\mathrm{Xu}, \mathrm{P} ., \mathrm{Yu}, \mathrm{B}$., Yun, M., et al. Heat conduction in fractal treelike branched networks. Int. J. Heat Mass Transf. 2006, 49(19-20): 3746-3751.

Yang, S., Liang, M., Yu, B., et al. Permeability model for fractal porous media with rough surfaces. Microfluid. Nanofluidics 2015, 18(5): 1085-1093.

Yang, S., Yu, B., Zou, M., et al. A fractal analysis of laminar flow resistance in roughened microchannels. Int. J. Heat Mass Transf. 2014, 77: 208-217.

Yu, B., Cai, J., Zou, M. On the physical properties of apparent two-phase fractal porous media. Vadose Zone J. 2009, 8(1): 177-186.

Yu, B., Cheng, P. Fractal models for the effective thermal conductivity of bidispersed porous media. J. Thermophys Heat Trans. 2002a, 16(1): 22-29.

Yu, B., Cheng, P. A fractal permeability model for bi-dispersed porous media. Int. J. Heat Mass Transf. 2002b, 45(14): 2983-2993.

Zou, M., Yu, B., Cai, J., et al. Fractal model for thermal contact conductance. J. Heat Transfer 2008, 130(10): 101301. 